\title{
Chronic fluoxetine treatment increases daytime melatonin synthesis in the rodent
}

This article was published in the following Dove Press journal:

Clinical Pharmacology:Advances and Applications

4 September 2009

Number of times this article has been viewed

\section{Gillian W Reierson \\ Claudio A Mastronardi \\ Julio Licinio \\ Ma-Li Wong}

Center on Pharmacogenomics, Department of Psychiatry and Behavioral Sciences, University of Miami Miller School of Medicine, Miami, FL, USA
Correspondence: Ma-Li Wong

Center on Pharmacogenomics,

Department of Psychiatry and Behavioral

Sciences, University of Miami Miller

School of Medicine, I580 NW I0th

Avenue, Bachelor Children's Research

Institute, Room 608, Miami, FL, USA

Tel $+6|26| 252550$

Fax +6I 261252337

Email mali.wong@anu.edu.au

\begin{abstract}
Circadian rhythm disturbances can occur as part of the clinical symptoms of major depressive disorder and have been found to resolve with antidepressant therapy. The pineal gland is relevant to circadian rhythms as it secretes the hormone melatonin following activation of the cyclic adenosine monophosphate (cAMP) signaling cascade and of arylalkylamine $\mathrm{N}$-acetyltransferase (AA-NAT), the rate-limiting enzyme for its synthesis. Cyclic AMP is synthesized by adenylate cyclases (AC) and degraded by phosphodiesterases (PDEs). Little is known about the contribution of the PDE system to antidepressant-induced alterations in pineal cAMP signaling and melatonin synthesis. In the present study we used enzyme immunoassay to measure plasma melatonin levels and pineal cAMP levels and as well as quantitative real-time polymerase chain reaction to measure pineal expression of PDE, AC, and AA-NAT genes in rats chronically treated with the prototypic antidepressant fluoxetine. We found elevated melatonin synthesis with increased pineal AA-NAT gene expression and daytime plasma melatonin levels and downregulated cAMP signaling with increased PDE and unchanged AC pineal gene expression, and decreased content of pineal cAMP. We conclude that chronic fluoxetine treatment increases daytime plasma melatonin and pineal AA-NAT gene expression despite downregulated pineal cAMP signaling in the rodent.
\end{abstract}

Keywords: antidepressant, melatonin, pineal, nucleotides, cyclic, phosphodiesterase, rat

\section{Introduction}

Multiple central nervous system (CNS) functions are dysregulated in major depressive disorder (MDD). Alterations in sleep, food intake, cognition, temperature, and neuroendocrine regulation are manifested as clinical symptoms in this chronic, highly disabling disorder. ${ }^{1}$ Clinical symptoms point to several brain areas of relevance to MDD pathophysiology including the pineal gland, HPA axis (hypothalamus, pituitary, adrenal glands), prefrontal cortex, and hippocampus. Circadian biorhythms have been found to occur with each of the above CNS functions. ${ }^{2}$ Furthermore, MDD and other mood disorders are associated with disruptions in these rhythms that are normalized with chronic antidepressant treatment. ${ }^{3,4}$ MDD symptoms can vary through the 24-hour period, with more severe symptoms often occurring in the morning, consistent with a phase advance of circadian rhythms. ${ }^{5}$ Unfortunately, the mechanism by which repeated antidepressants operate to restore these circadian rhythm disturbances in MDD remains unclear.

The central clock or pacemaker in the brain, the suprachiasmatic nucleus ( $\mathrm{SCN})$, is located in the anterior hypothalamus and is responsible for the entrainment of behavioral and biochemical circadian rhythms. The SCN receives information from the environment in the form of 'zeitgebers' or synchronizing signals such as light. Light enters the retina submit your manuscript | www.dovepress.com

Dovepress
Clinical Pharmacology: Advances and Applications 2009:I I-6

(C) 2009 Reierson et al, publisher and licensee Dove Medical Press Ltd. This is an Open Access article which permits unrestricted noncommercial use, provided the original work is properly cited. 
and is encoded by retinal ganglion cells into a message that is sent to the SCN via the retinohypothalamic tract. The SCN then sends projections via the paraventricular nucleus of the hypothalamus to affect the autonomic nervous system, the release of hormones from the pituitary, and the release of melatonin from the pineal gland. Brainstem raphe nuclei send serotonin/5-HT projections to the SCN, therefore the 5-HT system has been implicated in circadian rhythm disturbances. ${ }^{6}$ The circadian secretion of melatonin by the pineal gland is under the influence of the SCN, with higher levels during at night (darkness) and lower levels during daytime (light), corresponding with the sleep-wake cycle. Levels of melatonin can cause a feedback regulation on the activity of the SCN by binding to melatonin type- 1 and type- 2 receptors (MT1 and MT2) that are present in high concentrations in the SCN. Melatonin binding to MT1 receptors causes inhibition and binding to MT2 receptors results in a phase-shifting effect on SCN activity. ${ }^{7}$ The melatonergic agonist and 5HT2C antagonist agomelatine is a chronobiotic that not only resynchronizes dysregulated circadian sleep patterns in MDD, but also demonstrates potential as a novel antidepressant, displaying superior efficacy versus placebo and similar efficacy versus other antidepressant drugs on depressive symptoms in several randomized controlled clinical trials thus far. ${ }^{8}$

The pineal gland can be used as a model system to study the effects of antidepressants on circadian rhythms, as signaling transduction cascades in the pineal have been extensively studied and the activation of these cascades is known to result in a well-defined output, the synthesis and secretion of melatonin. ${ }^{9}$ Dense sympathetic innervation from the superior cervical ganglion results in release of norepinephrine $(\mathrm{NE})$ that binds to $\beta 1$-adrenergic receptors $(\beta 1-A R)$ on pinealocytes. Stimulation of $\beta 1-A R$ leads to activation of the adenylate cycase (AC)/cyclic adenosine monophosphate (cAMP)/protein kinase A (PKA) signaling cascade, increased activity of the rate-limiting enzyme of melatonin synthesis arylalkylamine $\mathrm{N}$-acetyltransferase (AA-NAT), and subsequently increased melatonin synthesis and secretion into the blood.

Treatment with pharmacologic agents, such as antidepressants, that chronically increase synaptic NE and/or 5-HT would be expected to affect the levels of signaling cascade intermediates. The amount of extracellular NE and 5-HT at the synapse and the duration of $\beta 1-\mathrm{AR}$ and $5 \mathrm{HT}_{2} \mathrm{R}$ stimulation could therefore influence pinealocyte metabolism and melatonin synthesis and secretion. However, the consequences of chronically increased extracellular NE or 5-HT on pinealocyte metabolism remain unknown. ${ }^{9}$ Attenuated activation of intracellular signaling pathway might occur as a compensatory mechanism to stabilize pinealocyte metabolism and circadian rhythms. The compensatory decrease of the signaling cascade has been hypothesized to occur as a result of either receptor downregulation or decreased synthesis of second messenger intermediates. However, increased degradation of cyclic nucleotides could also regulate pinealocyte metabolism in response to antidepressants. Therefore, phosphodiesterases (PDEs) could play a role in mediating effects of chronic antidepressant treatment to restore dysregulated circadian rhythms by modulating levels of cyclic nucleotide intermediates in signaling transduction cascades.

The regulation of cyclic nucleotide levels occurs through the balance of production by cyclase enzymes and degradation by PDE enzymes. As second messengers in intracellular signaling transduction cascades, cyclic nucleotides can have important downstream consequences on gene expression and resultant neuronal responses. By modulating levels of cyclic nucleotides such as cAMP, the activity of PDE enzymes could potentially influence melatonin secretion, one such neuronal response in the pineal gland. In the present study, we have used enzyme immunoassay and quantitative real-time PCR to investigate changes in plasma melatonin, AA-NAT expression, $\mathrm{PDE}$ and $\mathrm{AC}$ expression, and cAMP levels in the pineal gland to determine the effects of chronic antidepressant treatment on the cAMP signaling and melatonin synthetic transduction cascades in the pineal gland.

\section{Materials and methods}

\section{Animals and drug treatment}

Virus- and antibody-free adult, male Sprague Dawley rats (150-200 g; Harlan, Indianapolis, IN) were housed two per cage at $24^{\circ} \mathrm{C}$ with lights on from 06:00 to 18:00 hours in a stress-free environment for at least five days before the initiation of experimental procedures. Ten rats were randomly assigned to each experimental group: 1) control $(0.9 \%$ saline) or 2$)$ fluoxetine (selective serotonin reuptake inhibitor). All animals received daily (at 10:00 am) 0.5 mL intraperitoneal (i.p.) injections of either $0.9 \%$ saline (Hospira Inc., Lake Forest, IL) or $5.0 \mathrm{mg} / \mathrm{kg}$ fluoxetine (Eli Lilly, Indianapolis, IN) dissolved in $0.9 \%$ saline for eight weeks. The $5.0 \mathrm{mg} / \mathrm{kg}$ dose for fluoxetine is based on previous reports. ${ }^{10}$ We have previously utilized the chronic time course of eight weeks daily i.p. antidepressant injections and do not report any indication of inflammation or stress as a result of the injections. Furthermore, eight weeks daily treatment with $5 \mathrm{mg} / \mathrm{kg}$ fluoxetine i.p. has been reported to decrease expression of the corticotropin-releasing hormone gene in the 
perviventricular nucleus of the hypothalamus, an effect that was not seen at two weeks. ${ }^{10}$ Therefore, eight weeks treatment was chosen as clinically antidepressants are effective after 3-6 weeks. ${ }^{10}$ Animals were euthanized by decapitation and the pineal gland was dissected out, flash frozen, and stored at $-80{ }^{\circ} \mathrm{C}$. Animals received their last treatment 24 hours before termination of experiments and were sacrificed during the daytime, between 10:00 am and 12:00 pm. We selected this light cycle timepoint, corresponding to four to six hours after the dark/light transition, to study the effect of antidepressant treatment at the nadir of circadian cycles for melatonin synthesis and cAMP signaling to increase the likelihood of detecting significant differences. This experimental protocol was approved by the University of Miami Institutional Animal Care and Use Committee.

\section{Enzyme immunoassays (EIA)}

Melatonin concentration in the plasma was determined using a competitive enzyme immunoassay kit (IBL Hamburg, Hamburg, Germany). Samples, standards, and controls were extracted, eluted, evaporated, and reconstituted; the test procedure was then performed as outlined in the manufacturer's protocol. The concentration of melatonin is expressed as $\mathrm{pg} / \mathrm{mL}$.

Cyclic AMP levels in the pineal gland were determined using a direct, competitive enzyme immunoassay kit (Assay Designs, Ann Arbor, MI). Pineal glands were homogenized in 10 volumes of $0.1 \mathrm{M} \mathrm{HCl}$ and centrifuged $\geq 600 \times \mathrm{g}$ at room temperature. The supernatant was acetylated to improve signal detection and samples were run in duplicate. The concentration of cAMP is expressed as $\mathrm{pmol} / \mathrm{mL}$.

\section{Quantitative real-time RT-PCR}

Total RNA was isolated from pineal gland using RNeasy Lipid Mini Kit, quantified using a Nanodrop ND-1000 spectrophotometer (Thermo-Fisher Scientific, Waltham, MA) and reverse transcribed to cDNA using Omniscript RT Kit (Qiagen, Hilden, Germany), random hexamer primers, and 250 ng RNA. Based on the results of a pilot study in which we surveyed all of the PDEs expressed in the pineal gland, we selected to follow up the results for PDE -4B, and $-8 \mathrm{~B}$ genes. PDE primers were designed in different exons to amplify cDNA using Primer Express software (Applied Biosystems, Foster City, CA). PDE4B (NM_017031) primers were: forward (5'-GTGACGAGCTCCGGTGTTC-3') and reverse (5'-GCCGATACAACTCCAAGGACTT-3'). ${ }^{11}$ PDE8B (NM_199268) primers were: forward (5'-GAAGCCGTGTGCAGGTCAAT-3') and reverse
( $5^{\prime}$ - A ACCACAGCCAGGATCACTGT- ${ }^{\prime}$ ). AC 1 (NM_001107239) primers were: forward (5'-CCCCGGAACATGGATCTCTATTA-3') and reverse (5'-TGTTGTTGCCATCCAGTTCTATG-3'). AA-NAT (U40803) primers were: forward (5'-GAAGGGAGACAGCAGTTC-3') and reverse (5'-GTCCTGGTCTTGCCTTTG-3'). ${ }^{12}$ Beta-Actin (NM_031144) primers were: forward (5'- GACCCAGATCATGTTTGAGACCTT-3') and reverse (5'-AGAGGCATACAGGACAACACA-3'). A standard curve of pooled, serially diluted cDNA was run for each target gene and for a housekeeping gene, rat $\beta$-Actin, using the 7900HT Fast Real-Time PCR System (Applied Biosystems). cDNA samples were diluted 1:250 and run in triplicate for both rat $\beta$-Actin and the gene of interest. The three SYBR cycle threshold $(\mathrm{Ct})$ values were averaged for each sample, and the RNA input for the target gene was calculated from the standard curve. Fold changes in pineal gland target gene expression was expressed as a ratio to $\beta$-Actin expression for each sample.

\section{Statistical analysis}

The data were analyzed using Student's $t$-test with the significance level set at $p<0.05$.

\section{Results}

\section{Enzyme immunoassays}

The levels of plasma melatonin were increased by chronic fluoxetine $(+39 \% ; p<0.05 ; \mathrm{t}=2.818, \mathrm{df}=17)$ (Figure $1 \mathrm{~b})$. Pineal gland cAMP levels were decreased by chronic fluoxetine $(-50 \% ; p<0.01 ; \mathrm{t}=3.229, \mathrm{df}=13)$ (Figure $2 \mathrm{~b})$.

\section{Quantitative real-time RT-PCR}

Chronic treatment with fluoxetine increased the gene for the rate-limiting enzyme of melatonin synthesis, AA-NAT $(+393 \% ; p<0.001 ; \mathrm{t}=4.440, \mathrm{df}=17)$ (Figure 1a).

Chronic fluoxetine increased the expression of two cAMP-specific PDEs, PDE4B $(+140 \% ; p<0.001$; $\mathrm{t}=4.077, \mathrm{df}=17)$ and PDE8B $(+68 \% ; p<0.01 ; \mathrm{t}=3.219$, $\mathrm{df}=18)$. Expression of the predominant adenylate cyclase isoform, $\mathrm{AC} 1$, was unchanged $(p>0.05 ; \mathrm{t}=0.1768$, $\mathrm{df}=16)($ Figure $2 \mathrm{a})$.

\section{Discussion}

In the present study, we provide evidence of increased daytime melatonin synthesis in the rodent following eight weeks sustained treatment with the prototypic antidepressant fluoxetine. In order to understand the effect of chronic treatment with fluoxetine on the melatonin synthetic cascade, we measured expression of the 
a)

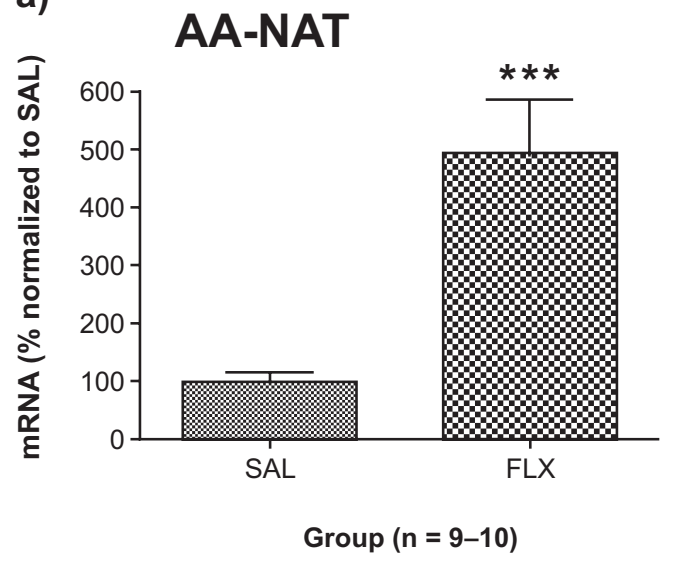

b)

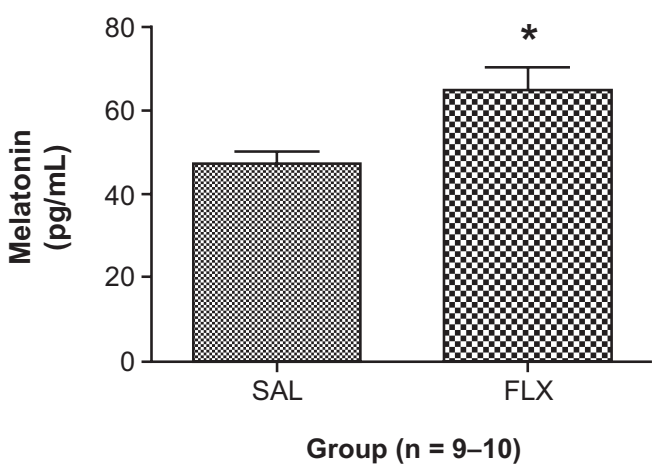

Figure I Effect of chronic FLX treatment on a) AA-NAT gene expression levels in the pineal gland and b) plasma melatonin levels. AA-NAT gene expression results are expressed as mean \pm SEM for FLX as percentage of SAL $(n=9-10$ animals/group). Melatonin enzyme immunoassay results are expressed as mean \pm SEM concentrations of plasma melatonin (in $\mathrm{pg} / \mathrm{mL}$ ) of rats treated chronically with $\mathrm{SAL}$ and $\mathrm{FLX}(\mathrm{n}=9$-10/group).

Note: Asterisks indicate significant between-group differences $\left({ }^{*} p<0.05\right.$, $\left.{ }^{* * *} p<0.00 \mathrm{I}\right)$ using Student's $t$-test.

Abbreviations: AA-NAT, arylalkylamine $\mathrm{N}$-acetyltransferase; FLX, fluoxetine; SAL; saline-treated control; SEM, standard error of mean.

AA-NAT gene in the pineal gland and found five-fold increased expression of AA-NAT following chronic fluoxetine. We then measured blood plasma melatonin using enzyme immunoassay to determine the consequences of increased AA-NAT gene expression in the pineal gland. We predicted that plasma melatonin levels would be elevated following chronic fluoxetine because of the findings of increased transcription of the gene for the rate-limiting enzyme of melatonin synthesis, AA-NAT. Plasma melatonin levels were increased following chronic fluoxetine. We took blood samples during the light cycle, when plasma melatonin levels are usually at their nadir, and were able to detect an increase. Our finding of increased plasma melatonin is in contrast with one study that found melatonin unaltered following chronic imipramine (IMI) ${ }^{13}$ and other studies that have found chronic treatment (10 days to three weeks) with monoamine oxidase inhibitors, ${ }^{13,14} \mathrm{IMI}^{15,16}$ desipramine, ${ }^{17}$ and venlafaxine ${ }^{18}$ decreased or attenuated increases in plasma or pineal melatonin, its precursors, and/or melatonin synthetic enzyme activity. We administered fluoxetine, a selective serotonin reuptake inhibitor, chronically for eight weeks, whereas other studies administered tricyclic antidepressants for 10 days to three weeks; therefore, differences in duration of treatment and/or mechanism of action at the synapse could account for this discrepancy. Other factors such as diet and animal age can impact tryptophan metabolism and therefore the effect of fluoxetine. As our animals received the same standard chow diet and were of similar age, it is unlikely these parameters influenced the effect of fluoxetine.

Having found an upregulation of melatonin synthesis, we then investigated whether chronic fluoxetine alters expression of upstream cAMP signaling cascade elements in the pineal gland, such as the PDE and cyclase genes. We found that chronic treatment with fluoxetine increased the expression of two cAMP-specific PDE genes, PDE4B and PDE8B, while the expression of the cAMP-specific synthetic enzyme, AC1, was unchanged. Despite evidence that the PDE4 inhibitor rolipram displays antidepressant effects both in humans ${ }^{19,20}$ and rodents, ${ }^{21,22} \mathrm{PDE} 4$ gene and protein expression is paradoxically increased after chronic antidepressant and electroconvulsive shock treatment in the cortex and hippocampus of rodents. ${ }^{23}$ In contrast to our findings of increased expression of cAMPspecific PDE isoforms, PDE4B and PDE8B, in the rat pineal following chronic treatment with fluoxetine, another study found that two weeks' treatment with $3 \mathrm{mg} / \mathrm{kg}$ fluoxetine via osmotic minipump decreased pineal PDE4B mRNA as measured by ISH. ${ }^{24}$ The animals in the present study were euthanized four to six hours into the light cycle (from 10:00 to 12:00); therefore, we have measured PDE gene expression during the light phase. PDE activity exhibits circadian rhythms in the rat pineal with highest levels during the dark phase and the lowest levels during the light phase..$^{25}$ The circadian rhythmicity of PDEs in the rat pineal also demonstrates sex differences, with two peaks of activity in male rats (from 2:00-4:00 am and again at 4:00 pm) versus only one peak (from 2:00-4:00 am) in females and castrated male rats. ${ }^{26} \mathrm{PDE} 4 \mathrm{~B}$ mRNA demonstrates a specific circadian rhythm, with peak levels at night in the rat pineal four hours into the dark phase that progressively decline into the light phase. PDE4B2 levels are five-fold greater at night versus the day. ${ }^{27}$ As we did not measure PDE gene expression at different circadian timepoints, we cannot specifically comment on chronic antidepressant induced alterations in the 
a)

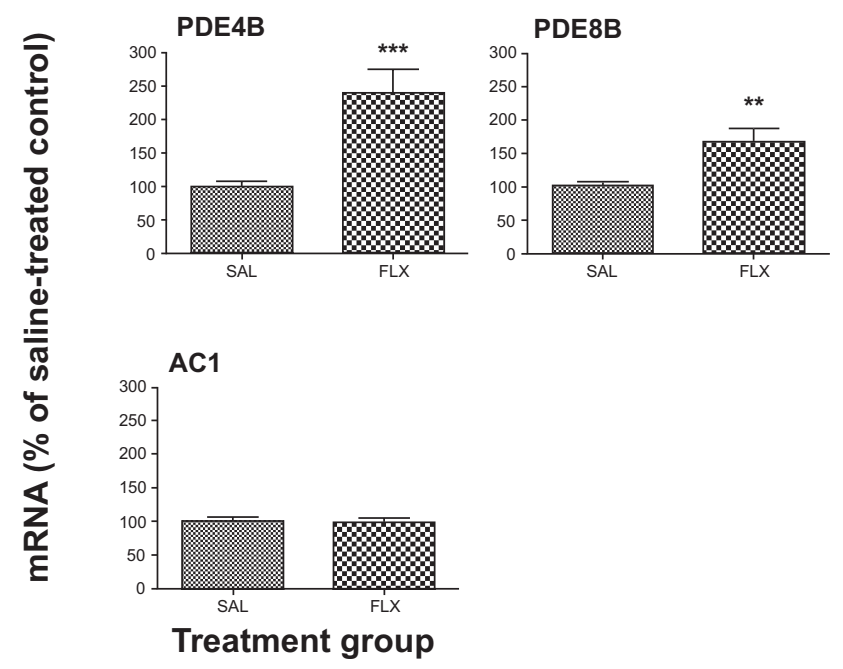

b)

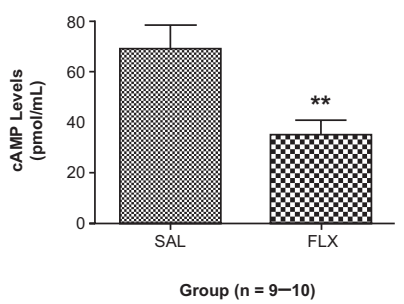

Figure 2 Effect of chronic FLX treatment on a) PDE and AC gene expression and b) CAMP levels in the rat pineal gland. Gene expression results are expressed as mean \pm SEM for FLX as percentage of saline-treated control $(S A L)(n=9-10$ animals $/$ group). Cyclic AMP (cAMP) enzyme immunoassay results are expressed as mean $\pm \mathrm{SEM}$ concentrations of cAMP (in pmol/mL) for SAL and FLX.

Note: Asterisks indicate significant differences $(* * p<0.01$, ***p $<0.00 \mathrm{I})$ using Student's $t$-test.

Abbreviations: AC, adenylate cyclases; AMP, adenosine monophosphate; FLX, fluoxetine; SAL; saline-treated control; PDE, phosphodiesterase; SEM, standard error of mean.

circadian rhythms of PDEs in the pineal gland. However, our finding of increased PDE4B expression during the day suggests that chronic fluoxetine treatment has altered the activity of the PDE system during its nadir, possibly causing a shift in the circadian rhythm.

To determine the consequence of altered PDE expression following chronic treatment with fluoxetine, we assayed the content of cAMP in the pineal gland using a competitive enzyme immunoassay. Since we found increased expression of cAMP-specific PDEs and unchanged expression of the cAMP-specific adenylate cyclase following chronic treatment, we predicted that cAMP levels would be decreased in the pineal gland chronically. Indeed, chronic fluoxetine was found to decrease pineal cAMP levels to half of the values seen in the saline group. These results are in agreement with our recent report of downregulated cAMP signaling in rats chronically treated with imipramine in which we found increased PDE, decreased AC, decreased cAMP levels, and unchanged cGMP levels. ${ }^{28}$ Similarly, in the pineal gland, congruent findings of increased PDE expression, unchanged AC, and decreased cAMP levels suggest that repeated treatment with fluoxetine decreases cAMP signaling in the rat pineal. It is unclear why cAMP signaling was downregulated despite an upregulation of melatonin synthesis as indicated by increased pineal AA-NAT expression and plasma melatonin levels. It is possible that the downregulation of cAMP signaling might occur as a compensatory adaptation to bring the increased melatonin synthetic cascade back to baseline levels.

Several lines of evidence in rodents point to an influence of SSRIs such as fluoxetine on circadian rhythms. Fluoxetine inhibits light-induced phase advances in circadian wheel running behavior, alters clock gene expression in the $\mathrm{SCN}$, and decreases REM sleep..$^{29-32}$ Our finding of chronic fluoxetine induced increases in daytime plasma melatonin is consistent with the idea that antidepressants might normalize the phase advance in circadian rhythms that occurs with MDD by causing a shift in the pattern of melatonin secretion. As circadian secretion of the hormone melatonin peaks at night and declines during the day, it is possible that chronic antidepressant treatment induces increases in daytime melatonin that could in turn affect sleep-wake cycles. The behavioral consequences of increased daytime melatonin are unclear, but possibly point to an improvement in the quality of sleep by decreasing early morning awakening, a common complaint in MDD. For example, melatonin supplements have been widely reported to have a sleep promoting effect when taken during the day, and therefore are used as a treatment for jet lag syndrome. ${ }^{33}$

In summary, chronic fluoxetine increased pineal AA-NAT expression and elevated daytime plasma melatonin despite decreased pineal cAMP content and increased pineal PDE gene expression. We conclude that repeated fluoxetine treatment increases melatonin synthesis in the rodent and alters pineal cAMP signaling.

\section{Disclosure}

Gillian W Reierson contributed to the conception and design of the study, the acquisition of data, the analysis and interpretation of data, and drafting of the article. Claudio A Mastronardi contributed to the conception and design of the study, the acquisition of data, and revising the article for important intellectual content. Julio Licinio contributed to revising the article for important intellectual content and final approval of the version of the article to be published. Ma-Li Wong contributed to the 
conception and design of the project, analysis and interpretation of data, revising the article for important intellectual content, and final approval of the article to be published. The authors report no conflicts of interest in this work.

\section{References}

1. Wong ML, Licinio J. Research and treatment approaches to depression. Nat Rev Neurosci. 2001;2(5):343-351.

2. McClung CA. Circadian genes, rhythms and the biology of mood disorders. Pharmacol Ther. 2007;114(2):222-232.

3. Bunney WE, Bunney BG. Molecular clock genes in man and lower animals: possible implications for circadian abnormalities in depression. Neuropsychopharmacology. 2000;22(4):335-345.

4. Lam RW. Sleep disturbances and depression: a challenge for antidepressants. Int Clin Psychopharmacol. 2006;21(Suppl 1):S25-S29.

5. Lader M. Limitations of current medical treatments for depression: disturbed circadian rhythms as a possible therapeutic target. Eur Neuropsychopharmacol. 2007;17(12):743-755.

6. Turek FW, Dugovic C, Zee PC. Current understanding of the circadian clock and the clinical implications for neurological disorders. Arch Neurol. 2001;58(11):1781-1787.

7. Srinivasan V, Pandi-Perumal SR, Trakht I, et al. Pathophysiology of depression: role of sleep and the melatonergic system. Psychiatry Res. 2009;165(3):201-214

8. Dolder CR, Nelson M, Snider M. Agomelatine treatment of major depressive disorder. Ann Pharmacother. 2008;42(12):1822-1831.

9. Simonneaux V, Ribelayga C. Generation of the melatonin endocrine message in mammals: a review of the complex regulation of melatonin synthesis by norepinephrine, peptides, and other pineal transmitters. Pharmacol Rev. 2003;55(2):325-395.

10. Brady LS, Gold PW, Herkenham M, Lynn AB, Whitfield HJ Jr. The antidepressants fluoxetine, idazoxan and phenelzine alter corticotropinreleasing hormone and tyrosine hydroxylase mRNA levels in rat brain: therapeutic implications. Brain Res. 1992;572(1-2):117-125.

11. Wang H, Edens NK. mRNA expression and antilipolytic role of phosphodiesterase 4 in rat adipocytes in vitro. J Lipid Res. 2007;48(5):1099-1107.

12. Spessert R, Gupta BB, Rohleder N, Gerhold S, Engel L. Cyclic AMP-inducible genes respond uniformly to seasonal lighting conditions in the rat pineal gland. Neuroscience. 2006;143(2):607-613.

13. Ask AL, Fowler CJ, Hall H, Kelder D, Ross SB, Saaf J. Cortical beta- and alpha 2- adrenoceptor binding, hypothalamic noradrenaline and pineal melatonin concentrations measured at different times of the day after repeated treatment of rats with imipramine, zimeldine, alaproclate and amiflamine. Acta Pharmacol Toxicol (Copenh). 1986;58(1):16-24.

14. Oxenkrug GF, Requintina PJ, McIntyre IM, White K. Chronic effect of the irreversible and reversible selective MAO-A inhibitors on rat pineal melatonin biosynthesis. J Neural Transm Suppl. 1994;41:381-384.

15. Friedman E, Yocca FD, Cooper TB. Antidepressant drugs with varying pharmacological profiles alter rat pineal beta adrenergic-mediated function. J Pharmacol Exp Ther. 1984;228(3):545-550.

16. Friedman E, Cooper T, Yocca F. The effect of imipramine treatment on brain serotonin receptors and beta-adrenoceptors and on pineal beta-adrenergic function in adult and aged rats. Eur J Pharmacol. 1986;123(3):351-356.

Clinical Pharmacology: Advances and Applications

\section{Publish your work in this journal}

Clinical Pharmacology: Advances and Applications is an international, peer-reviewed, open access journal publishing original research, reports, reviews and commentaries on all areas of drug experience in humans. The manuscript management system is completely online and includes a very quick and fair peer-review system, which is all easy to use.
17. Heydorn WE, Brunswick DJ, Frazer A. Effect of treatment of rats with antidepressants on melatonin concentrations in the pineal gland and serum. J Pharmacol Exp Ther. 1982;222(3):534-543.

18. Franklin M, Clement EM, Campling G, Cowen PJ. Effect of venlafaxine on pineal melatonin and noradrenaline in the male rat. J Psychopharmacol. 1998;12(4):371-374.

19. Zeller E, Stief HJ, Pflug B, Sastre-y-Hernandez M. Results of a phase II study of the antidepressant effect of rolipram. Pharmacopsychiatry. 1984;17(6):188-190.

20. Bobon D, Breulet M, Gerard-Vandenhove MA, et al. Is phosphodiesterase inhibition a new mechanism of antidepressant action? A double blind double-dummy study between rolipram and desipramine in hospitalized major and/or endogenous depressives. Eur Arch Psychiatry Neurol Sci. 1988;238(1):2-6.

21. Zhang HT, Huang Y, Jin SL, et al. Antidepressant-like profile and reduced sensitivity to rolipram in mice deficient in the PDE4D phosphodiesterase enzyme. Neuropsychopharmacology. 2002;27(4):587-595.

22. Itoh T, Tokumura M, Abe K. Effects of rolipram, a phosphodiesterase 4 inhibitor, in combination with imipramine on depressive behavior, CRE-binding activity and BDNF level in learned helplessness rats. Eur J Pharmacol. 2004;498(1-3):135-142.

23. Esposito K, Reierson GW, Rong Luo H, Sheng Wu G, Licinio J, Wong ML. Phosphodiesterase genes and antidepressant treatment response: A review. Ann Med. 2009;41(3):177-185.

24. Miro X, Perez-Torres S, Artigas F, Puigdomenech P, Palacios JM, Mengod G. Regulation of cAMP phosphodiesterase mRNAs expression in rat brain by acute and chronic fluoxetine treatment. An in situ hybridization study. Neuropharmacology. 2002;43(7):1148-1157.

25. Minneman KP, Iversen LL. Diurnal rhythm in rat pineal cyclic nucleotide phosphodiesterase activity. Nature. 1976;260(5546):59-61.

26. Epplen JT, Kaltenhauser H, Engel W, Schmidtke J. Patterns of cyclic AMP phosphodiesterases in the rat pineal gland: sex differences in diurnal rhythmicity. Neuroendocrinology. 1982;34(1):46-54.

27. Kim JS, Bailey MJ, Ho AK, Moller M, Gaildrat P, Klein DC. Daily rhythm in pineal phosphodiesterase (PDE) activity reflects adrenergic/3', 5'-cyclic adenosine 5'-monophosphate induction of the PDE4B2 variant. Endocrinology. 2007;148(4):1475-1485.

28. Reierson GW, Mastronardi CA, Licinio J, Wong ML. Chronic imipramine downregulates cyclic AMP signaling in rat hippocampus. Neuroreport. 2009;20(3):307-311.

29. Gannon RL, Millan MJ. Evaluation of serotonin, noradrenaline and dopamine reuptake inhibitors on light-induced phase advances in hamster circadian activity rhythms. Psychopharmacology (Berl). 2007; 195(3):325-332.

30. Cuesta M, Clesse D, Pevet P, Challet E. New light on the serotonergic paradox in the rat circadian system. J Neurochem. 2009;10(1): 231-243.

31. Cuesta M, Mendoza J, Clesse D, Pevet P, Challet E. Serotonergic activation potentiates light resetting of the main circadian clock and alters clock gene expression in a diurnal rodent. Exp Neurol. 2008;210(2):501-513.

32. Ivarsson M, Paterson LM, Hutson PH. Antidepressants and REM sleep in Wistar-Kyoto and Sprague-Dawley rats. Eur J Pharmacol. 2005;522(1-3):63-71.

33. Arendt J. Melatonin: characteristics, concerns, and prospects. J Biol Rhythms. 2005;20(4):291-303.

Visit http://www.dovepress.com/testimonials.php to read real quotes from published authors. 